\title{
Utilization of idle time slot in spectrum sensing under noise uncertainty
}

\author{
Iyad Khalil Tumar', Adnan Mohammad Arar², Ayman Abd El-Saleh ${ }^{3}$ \\ ${ }^{1}$ Electrical and Computer Engineering Department, Faculty of Engineering and Technology, Birzeit University, Ramallah, Palestine \\ ${ }^{2}$ Department of Mathematics, College of Science and Technology, Hebron University, Hebron, Palestine \\ ${ }^{3}$ Department of Electronics and Communication Engineering, College of Engineering, A'Sharqiyah University, Ibra, Oman
}

\begin{abstract}
Article Info
Article history:

Received Mar 2, 2021

Revised Jul 13, 2021

Accepted Jul 28, 2021

\section{Keywords:}

Asynchronous sensing

Cognitive radio

Noise uncertainty

SNR wall

ABSTRACT

Spectrum sensing in cognitive radio (CR) is a critical process as it directly influences the accuracy of detection. Noise uncertainty affects the reliability of detecting vacant holes in the spectrum, thus limiting the access of that spectrum by secondary users (SUs). In such uncertain environment; SUs sense the received power of a primary user (PU) independently with different measures of signal-to-noise ratio (SNR). Long sensing time serves in mitigating the effect of noise uncertainty, but on the cost of throughput performance of CR system. In this paper, the scheme of an asynchronous and crossed sensing-reporting is presented. The scheme reduces energy consumption during sensing process without affecting the detection accuracy. Exploiting the included idle time $\left(T_{i}\right)$ in sensing time slot; each SU collects power samples with higher SNR directly performs the reporting process to a fusion center (FC) consecutively. The FC terminates the sensing and reporting processes at a specific sensing time that corresponds to the lowest SNR $\left(S N R_{\text {wall }}\right)$. Furthermore, this integrated scheme aims at optimizing the total frame duration $\left(T_{f}\right)$. Mathematical expressions of the scheme are obtained. Analytical results show the efficiency of the scheme in terms of energy saving and throughput increment under noise uncertainty.
\end{abstract}

This is an open access article under the CC BY-SA license.

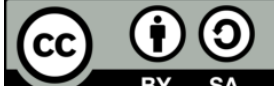

\section{Corresponding Author:}

Adnan Mohammad Arar

College of Science and Technology, Hebron University

Hebron, Palestine

Email: adnana@hebron.edu

\section{INTRODUCTION}

Opportunistic cognitive radio aims at making the radio spectrum access more efficient, thus resolving the spectrum underutilization problem of traditional wireless communication systems. This is attained through allowing unlicensed secondary users (SUs) to exploit free licensed frequency bands under quality of service (QoS) constraints of licensed primary users (PUs). SUs monitor the primary signal through spectrum sensing to avoid interfering with the PUs [1]. Once a spectrum hole is sensed, the SUs are allowed to opportunistically access it. Therefore, sensing the licensed spectrum is envisioned as a critical initial stage towards the development of practical cognitive radio (CR) systems [2], [3].

$\mathrm{CR}$ is the core technology behind spectrum reuse in which the SUs automatically sense the spectrum and make efficient use of any available slots at any given time. In a wider sense, CR systems allow unlicensed users to sense, access and use licensed spectrums of licensed users by dynamically changing its parameters such as power and data rate. These parameter changes of CR systems depend on the ever changing operational environment as a result of temporal, spatial and spectral variations of licensed users' activities. It is practically viable to assume that the SUs don't have any prior information about the primary 
signal [4], thus, the method of energy detection (ED) in spectrum sensing is wildly used and will be considered in this paper. In ED, a predefined detection threshold is determined to be within the average value of the noise power in communication environments [5]. The ED compares the received energy on a primary band with the threshold during the sensing time duration, then, builds a decision on the presence or absence of a hole [1], [6].

The statistical measurements of the received signal power is generally governed by environmental constrains such as the signal-to-noise ratio (SNR) and judged based on the predefined detection threshold. Spectrum sensing is performed to enhance the efficiency of accessing the spectrum by SUs without causing harmful interference to PUs [7]. SUs' spectrum efficiency is achieved through transmitting data on the sensed free channels. Power efficiency is another major challenge that faces operators due to limited power resources. In opportunistic CR systems, there are three main processes consume energy, namely sensing the spectrum, reporting local decisions and transmitting data. If the frame structure of an opportunistic CR system is fixed, increasing the sensing and reporting time slots will be at the expense of spectrum usage efficiency. On the other hand, long SU transmission slots may violate QoS requirements since spectrum sensing is prohibited during this period, and a new PU may try to access its band while being used by SUs.

To evaluate the spectrum sensing performance at the receiver of the $i^{\text {th }} \mathrm{SU}$, two performance metrics are used [1], [8]. First, the false alarm probability $\left(P_{f, i}\right)$ which represents the likelihood of locally and individually deciding the absence of a hole whereas the hole is actually present. Second, the detection probability $\left(P_{d, i}\right)$ which refers to the probability of locally and individually deciding the presence of a hole while the hole is actually present [9], [10]. The two complements of the aforementioned probabilities are the correct detection $\left(P_{c, i}\right)$ and missed detection $\left(P_{m, i}\right)$ probabilities. $P_{c, i}$ denotes the probability of locally and individually decide the presence of a hole while the hole is actually present. Whereas $P_{m, i}$ refers to the probability of locally and individually decide the presence of a hole whereas the hole is actually absent. To achieve acceptable PU detection accuracy and protection against interference under noise uncertainty environment, a minimum $P_{d, i}$ should not be tolerated. On the other hand, a maximum $P_{f, i}$ should not be tolerated so as to attain as much chances to use the spectrum as possible. These intolerable minimum $P_{d, i}$ and maximum $P_{f, i}$ must be set to be as high and as low as possible, respectively.

Althunibat et al. [10] considered targeted performance metrics to reduce the consumed energy. In research [11]-[13], the authors made an assumption that considers the primary signal received by all SUs is with the same SNR. Liu et al. [14] aimed to reduce the energy consumption by considering an intrinsic tradeoff between spectrum and energy efficiencies in a CR network. Hamdi and Letaief [15] adopted a threshold constraint to minimize the transmission power and maximize the throughput. All these studies consider fixed sensing time slot for all SUs. In practical deployment, wireless communication environment suffers from noise uncertainty due to different internal and external factors [1]. Hence, various SUs receive the primary signal with different SNR values. To overcome noise uncertainty and satisfy the QoS conditions, cooperative spectrum sensing can be adopted. Besides, setting the detection threshold SUs' receivers should carefully accommodate such uncertain wireless environments so as to mitigate potential wrong noise variance estimation [1].

In this paper, enhancing the power efficiency of a CR system under noise uncertainty is considered. The main idea is to exploit a part of sensing time slot for reporting local decisions, and increase the transmission time slot within the total frame duration, while maintaining the detection metrics within acceptable ranges. This is done by utilizing an idle time period during the sensing time slot for those SUs who built their local decisions earlier. Due to noise uncertainty, it is assumed that every SU receives the primary signal with a different SNR value within a specific uncertainty range [16]. During spectrum sensing, the SU node that receives a strong signal can terminate constructing the local decision earlier than the others. Then, it sequentially performs reporting local decision to the fusion center (FC) by using asynchronous sensing scheme. Furthermore, SUs who receive signal with very low SNR (below $S N R_{\text {wall }}$ ) will not be allowed to perform the reporting process. This increases the detection performance under noise uncertainty and enhances the power efficiency. The required sensing time for the $i^{\text {th }} \mathrm{SU}$ is defined as a function of $S N R_{i}$ together with a targeted and predefined performance metrics; $P_{c, i}$ and $P_{m, i}$. Due to noise uncertainty, minimum acceptable detection probability and maximum permissible missed detection probability are targeted. Thus, power consumption during the sensing process is enhanced while shortening the global idle time, which in turn increases the transmission time and enhances the SUs' throughput as well. Also, SUs with low SNR are prevented from reporting their local decisions according to the maximum sensing time allowed, which corresponds to the $S N R_{\text {wall }}$. Numerical results are validated analytically. Energy and throughput efficiencies are examined and compared with classical schemes. 
The rest of this paper is organized as follows: Section 2 describes the system model of the CR network. Section 3 presents detailed analyses of the proposed scheme. Analytical results are discussed in section 4. Section 5 concludes the paper.

\section{SYSTEM MODEL}

Consider a centralized and opportunistic CR network with $M$ unlicensed SU mobile terminals. All SUs are included in the coverage area of a FC as shown in Figure 1. The communication environment suffers from noise uncertainty. Therefore, variant SUs receive the primary signal with different SNR values. The ED technique for spectrum sensing is considered with no prior information is available about the primary signal. To protect the primary signal against interference, all SUs sense the spectrum during a specific time duration $\left(T_{S}\right)$. The detection threshold $(\lambda)$ is determined in such a way that the SU can sense the occupancy of a PU under a specific zone of noise uncertainty even at low SNR [17], [18]. The uncertainty of noise is randomly distributed in a single interval as $\sigma_{w, i}^{2} \in\left[\Delta^{-1} \sigma_{w}^{2}, \Delta \sigma_{w}^{2}\right]$, where $\sigma_{w}^{2}$ is the noise power variance, $\Delta$ is a parameter that determines the uncertainty zone, $\Delta>1$, and $\lambda$ should be selected to be out of uncertainty zone [9], [19].

Local decisions about the channel state can be made by each node using a binary hypothesis testing problem. $H_{1}$ chosen in case the primary user is present and $H_{o}$ when the primary user is absent [20]. The sensing problem for each detector is formulated as [21], [22].

$$
\left\{\begin{array}{ll}
H_{0}: X_{i}[n]=W_{i}[n] & , \quad i \in[1 . . M], n \in[1 . . N] \\
H_{1}: X_{i}[n]=W_{i}[n]+S[n], & i \in[1 . . M], n \in[1 . . N]
\end{array}\right\}
$$

Where $X_{i}[n]$ denote the $n^{\text {th }}$ received sample by the $i^{\text {th }} \mathrm{SU}$, and $n=1,2, \ldots, N, i=1,2, \ldots, M . W_{i}[n]$ is the noise received by the $i^{\text {th }} \mathrm{SU}$ and $S[n]$ is the primary user signal. The $i^{\text {th }}$ SU collects $N$ energy samples from the sensed spectrum at the sampling frequency $f_{s}$ and during the sensing time frame $T_{s}$, and $N=T_{s} f_{s}$. At the ED of the $i^{\text {th }} \mathrm{SU}$ receiver, the local decision statistic $Y_{i}$ is estimated by $Y_{i}=\frac{1}{N} \sum_{n=1}^{N}\left|X_{i}(n)\right|^{2}[1]$. Then, the local decision about the channel state is formulated as a binary decision $D_{i}(0,1)$ by comparing $Y_{i}$ with a predefined $\lambda$ as [1].

$$
D_{i}= \begin{cases}1, & \text { if } Y_{i} \geq \lambda \\ 0, & \text { if } Y_{i}<\lambda\end{cases}
$$

Hence, if $D_{i}=0$, then the $i^{\text {th }}$ SU decides a presence of a free channel. Otherwise, the spectrum is assumed to be used by a PU. Both the noise and the primary signal powers are assumed to be (i.i.d) Gaussian-distributed random variables, with zero mean $\left(\mu_{o}\right)$ and variance $\sigma_{w, i}^{2}$ and $\sigma_{s}^{2}$, respectively. $\sigma_{m, i}^{2}=\sigma_{w, i}^{2}+\sigma_{s}^{2}$ being the sum of the noise and signal power received at the $i^{\text {th }} \mathrm{SU}$. The primary signal received by the $i^{\text {th }} \mathrm{SU}$ with $\mathrm{SNR}, \gamma_{i}=\frac{\sigma_{s}^{2}}{\sigma_{w, i}^{2}}[23]$. The performance of the $i^{\text {th }} \mathrm{ED}$ is estimated by using performance metrics $P_{f, i}, P_{d, i}, P_{c, i}$ and $P_{m, i}$ probabilities under the two hypotheses $H_{0}$ and $H_{1}$ as [10], [24].

$$
\begin{aligned}
& P_{f, i}=\operatorname{Pr}\left\{Y_{i} \geq \lambda \mid H_{0}\right\} \\
& P_{d, i}=\operatorname{Pr}\left\{Y_{i} \geq \lambda \mid H_{1}\right\} \\
& P_{c, i}=\operatorname{Pr}\left\{Y_{i}<\lambda \mid H_{0}\right\} \\
& P_{m, i}=\operatorname{Pr}\left\{Y_{i}<\lambda \mid H_{1}\right\}
\end{aligned}
$$

Performance metrics are calculated by integrating the PDF function of the chi-square distributions with $2 \mathrm{~N}$ degrees of freedom for the collected power under $H_{0}$ and $H_{1}$, respectively [1]. Due to uncertainty of the proposed communication environment, a large number of samples is used [25]. Thus and through the central limit theorem, the chi-square distribution is approximated as a Gaussian distribution random variable [23]. Hence, the PDF of $Y_{i}$ under $H_{0}$ and $H_{1}$ can be approximated as [26], [27].

$$
\left\{\begin{array}{l}
H_{0}: Y_{i} \sim \operatorname{Normal}\left(\sigma_{w, i}^{2}, \frac{2}{N} \sigma_{w, i}^{4}\right) \\
H_{1}: Y_{i} \sim \operatorname{Normal}\left(\sigma_{m, i}^{2}, \frac{2}{N} \sigma_{m, i}^{4}\right)
\end{array}\right\}
$$

Hence, $P_{f, i}$ and $P_{d, i}$ are given by [10]. 


$$
\begin{aligned}
& P_{f, i}=Q\left(\frac{\lambda-\sigma_{w, i}^{2}}{\sigma_{w, i}^{2} \sqrt{2 / N}}\right) \\
& P_{d, i}=Q\left(\frac{\lambda-\sigma_{m, i}^{2}}{\sigma_{m, i}^{2} \sqrt{2 / N}}\right)=Q\left(\frac{\lambda-\sigma_{w, i}^{2}\left(1+\gamma_{i}\right)}{\sigma_{w, i}^{2}\left(1+\gamma_{i}\right) \sqrt{2 / N}}\right)
\end{aligned}
$$

The classical frame structure is depicted in Figure 2(a), according to this; all SUs start sensing the spectrum simultaneously and terminate sensing after $T_{S}$ seconds. Then, each SU starts reporting the local decision during its reporting time slot $\left(T_{r}\right)$ sequentially, and it waits for the other SUs to report their decisions to the FC during an idle time $\left(T_{i}\right)$. Hence, the total reporting time duration spent is $M T_{r}$ seconds while the total idle time during one frame duration $\left(T_{f}\right)$ is $\left(M^{2}-M\right) T_{r}$ seconds. The remaining time slot is reserved for transmitting data in case the final decision concludes a presence of a free band or the PU signal is missdetected. The transmission time denoted as $T_{t}$ is then given by $T_{t}=T_{f}-T_{s}-M T_{r}$. Based on this frame structure, each SU may exercise four possible activities during the sensing, idle, reporting and transmission stages. In the sensing stage, the consumed power $\left(p_{s}\right)$ during $T_{s}$ is the received power. In the idle stage, the $\mathrm{SU}$ is assumed to be asleep during $T_{i}$, hence no power is consumed during this time slot. The SU consumes most of the energy during the reporting stage; this power is denoted as $\left(p_{r}\right)$. The remaining time of the frame duration is for data transmission in which the consumed power is $\left(p_{t}\right)$. Since the energy is defined as the product of consumed power by time, the total consumed energy during each frame duration by the $M$ SUs is given by [28],

$$
E_{t o t}=M\left(p_{s} T_{s}+p_{r} T_{r}+P_{u} p_{t} T_{t}\right)
$$

where $P_{u}$ represents the probability of having the final decision at the FC of unused, and given as

$$
P_{u}=P_{0} P_{C}+P_{1} P_{M}
$$

where $P_{0}$ is the probability of that the sensed spectrum is free, and $P_{1}=1-P_{0} . P_{C}$ and $P_{M}$ represent the overall correct and missed alarm probabilities according to the implemented fusion rule at the FC.

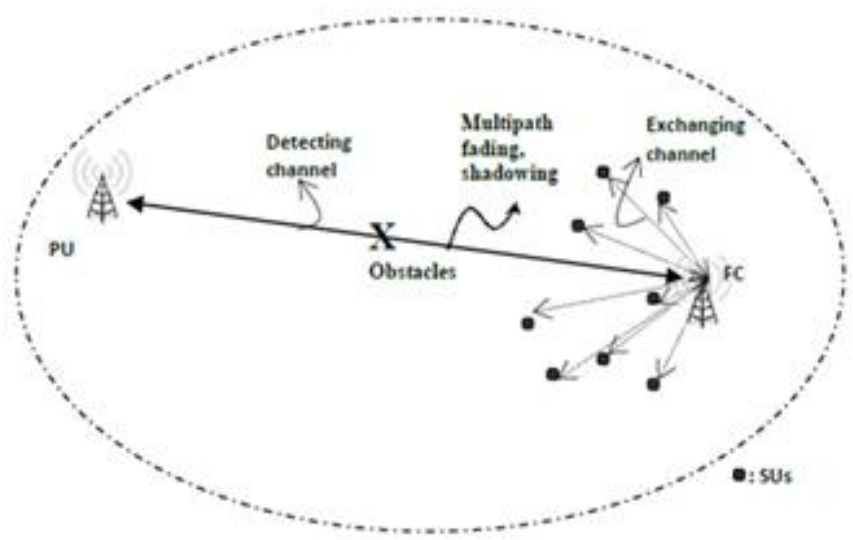

Figure 1. Centralized cognitive radio network characterized with noise uncertainty

\section{PROPOSED SCHEME}

Noise uncertainty is a phenomenon in which the noise variance fluctuates along the time and location dimensions due to different internal and external factors in the wireless communication environments. Hence, the sensing process in such environment is considered as the first critical process in CR systems. Because of this phenomenon, there exists a so called $S N R_{\text {wall }}$ which is defined as the SNR value below which the targeted detection performance cannot be achieved regardless of the sensing time [18]. As such, the performance parameters of spectrum sensing should be chosen carefully to accommodate a targeted performance metrics and to reduce the influence of noise uncertainty. The main aim of the proposed scheme is three-fold: 
- $\quad$ To develop an asynchronous sensing process in which a crossed sensing-reporting time slot, based on the SNR level for each SU, is proposed. The SUs utilize an idle time within the sensing time slot for reporting local decisions to the FC. Based on the $S N R_{\text {wall }}$, the number of SUs who are allowed to perform the crossed sensing-reporting process is identified, and the sensing time for each $\mathrm{SU}$ is governed by $\gamma_{i}$ of the corresponding node.

- $\quad$ To limit the total frame duration as compared to classical schemes so as to keep the SUs aware of any sudden changes in the channel occupancy.

- $\quad$ To reduce the consumed energy during the spectrum sensing process, and to increase the CR system efficiency by increasing the transmission time slot.

\subsection{Asynchronous sensing}

In classical schemes, all SUs perform spectrum sensing synchronously and terminate this activity once a specific time duration $T_{S}$ is elapsed [29]. Then, they start reporting their local decisions to the FC sequentially. In the proposed scheme, the detection and false alarm probabilities are chosen such that $\bar{P}_{d, i}=f\left(\gamma_{i}, \lambda, N_{i}\right) \geq P_{d, i}^{t h}$, and $\bar{P}_{f, i}=f\left(\lambda, N_{i}\right) \leq P_{f, i}^{t h}$, where $P_{d, i}^{t h}$ and $P_{f, i}^{t h}$ represent a minimum acceptable local detection probability and a maximum tolerated local false alarm probability, respectively [30]. To ensure that the sensing process is reliable, all nodes should achieve the same performance. Hence, $\bar{P}_{d, 1}=$ $\bar{P}_{d, 2}=\cdots=\bar{P}_{d}$ and $\bar{P}_{f, 1}=\bar{P}_{f, 2}=\cdots=\bar{P}_{f}$. This leads to adopt different sensing times due to different $\gamma_{i}$ for different SU [31].

To keep the PU well protected against interference and accommodate such uncertain environment, the OR fusion rule is employed at the FC to facilitate cooperative spectrum sensing. In the OR rule, the final decision at the FC will be "1" if at least one SU decides ' 1 '. Since the sensing time is inversely proportional with SNR, the FC terminates sensing process after a pre-defined time slot $\bar{T}_{s}^{\text {th }}$; where $\bar{T}_{S}^{\text {th }}$ represents the maximum sensing time that satisfies the targeted performance metrics and corresponds the $S N R_{\text {wall }}$. After the first sensing cycle, SUs which have not terminated their sensing processes are discarded from performing the reporting process. Therefore, $m$ out of $M$ SUs will still be performing the crossed sensing-reporting process. Hence, the overall correct probability for $M$ users, constrained by the local detection probability, $\bar{P}_{d}$, is given as (9):

$$
\bar{P}_{C}=\prod_{i=1}^{M}\left[Q\left(-Q_{d}^{-1}\left(1+\gamma_{i}\right)-\frac{\gamma_{i}}{\sqrt{2 / N_{i}}}\right)\right]
$$

and for $m$ users it is given as (10),

$$
\overline{\bar{P}}_{C}=\prod_{i=1}^{m}\left[Q\left(-Q_{d}^{-1}\left(1+\gamma_{i}\right)-\frac{\gamma_{i}}{\sqrt{2 / N_{i}}}\right)\right]
$$

where $Q$ represents the $Q$ function and $Q_{d}^{-1}$ represents the inverse $Q$ function of $\bar{P}_{d}, N_{i}$ is the number of collected samples by the $i^{\text {th }} \mathrm{SU}$ [30], [32]. During the sensing time frame, the SU that senses strong signals and satisfies the targeted correct probability consumes a shorter sensing time than that with a lower SNR [33], [34]. In this manner, each SU which has just terminated the sensing process can immediately start reporting the local decision to the FC and no need to enter into an idle period until other SUs terminate their sensing processes. By solving (9) and (10) separately, each SU consumes sensing time, $\bar{T}_{s, i}$ and $\overline{\bar{T}}_{s, i}$ for $M$ and $m$ users, respectively. Hence, $\bar{T}_{s, i}$ and $\overline{\bar{T}}_{s, i}$ that accommodate performance metrics and as a function of $\gamma_{i}$ can be expressed as (11),

$$
\begin{aligned}
& \bar{T}_{s, i}=\frac{2}{f_{s} \gamma_{i}^{2}}\left[-Q^{-1}\left(\bar{P}_{C}^{\frac{1}{M}}\right)-Q_{d}^{-1}\left(1+\gamma_{i}\right)\right]^{2} \\
& \overline{\bar{T}}_{s, i}=\frac{2}{f_{s} \gamma_{i}^{2}}\left[-Q^{-1}\left(\bar{P}_{C}^{\frac{1}{m}}\right)-Q_{d}^{-1}\left(1+\gamma_{i}\right)\right]^{2}
\end{aligned}
$$

Equation (11) and (12) show that the time needed for the sensing process is exponentially decreasing with $\gamma_{i}$. For $M$ users, the total time during crossed sensing-reporting process $\left(\bar{T}_{s r}\right)$ equals to $M \bar{T}_{s, i m a x}+T_{r}$, where $\bar{T}_{s, \text { imax }}=T_{s}$. For $m$ users, $\overline{\bar{T}}_{s r}=m \overline{\bar{T}}_{s, \text { imax }}+T_{r}$, where $\overline{\bar{T}}_{s, \text { imax }}$ represents the longest sensing time 
which corresponds to the sensing time consumed by the SU with SNR closed to $S N R_{\text {wall }}$, and $\overline{\bar{T}}_{s, i m a x} \leq \bar{T}_{S}^{t h}$. Hence, the total idle time duration $\left(\bar{T}_{i}\right)$ for $M$ SUs in one frame duration is (13),

$$
\bar{T}_{i}=M \bar{T}_{s, \text { imax }}-\sum_{i=1}^{M-1}\left(\bar{T}_{s, i}+T_{r}\right)
$$

and the total idle time duration $\left(\overline{\bar{T}}_{i}\right)$ for $m$ SUs in one frame duration is (14),

$$
\overline{\bar{T}}_{i}=m \overline{\bar{T}}_{s, \text { imax }}-\sum_{i=1}^{m-1}\left(\overline{\bar{T}}_{s, i}+T_{r}\right)
$$

The frame duration of the proposed crossed sensing-reporting process is depicted in Figure 2(b) and (c).

\subsection{Frame duration}

Typically, the total frame duration $T_{f}$ is divided into three main time slots; sensing, reporting and transmission time slots. In classical schemes, sensing time slot is usually predefined with a fixed duration [10]. After the sensing process terminated, SUs start reporting local decisions to the FC sequentially. Hence, the reporting time slot increases as the number of SUs increases [35], [36]. Then, the remaining time $T_{t}$ is allocated for data transmission, hence, $T_{f}=T_{s}+M T_{r}+T_{t}$, and the total consumed time by all SUs during one frame duration is $M T_{f}$. During the $T_{t}$ slot, SUs remain unaware of any sudden changes of channel occupancy [33]. Hence, the contradiction in $T_{t}$ setting should be taken into account. This is because allocating short $T_{t}$ leads to insufficient spectrum utilization from SUs' side, where the normalized throughput equals to $\frac{T_{t}}{T_{f}} P_{o} P_{c}$. On the other hand, allocating long $T_{t}$ will increase the possibility of collision between SUs and PU, which makes the PU less protectable against interference. In the previous subsection, a crossed sensing-reporting time slot is proposed in which a portion of the idle time during sensing process is utilized for reporting. This results in increasing the transmission time within the total frame duration. Hence, optimizing the frame duration will enable us to overcome the aforementioned critical contradiction in $T_{t}$ setting. Also, adopting high correct probability will contribute in reducing the collision probability and increasing the CR throughput. The total frame duration can be optimized to satisfy the condition $\bar{P}_{C}=f\left(\lambda, \bar{T}_{s, i}\right)>\bar{P}_{C}^{t h}$ which is constrained by $\bar{P}_{m}=f\left(\gamma_{i}, \lambda, \bar{T}_{s, i}\right)<\bar{P}_{m}^{t h}$, where $\bar{P}_{C}^{t h}$ is the minimum correct probability at which the throughput is the same as in classical schemes, and $\bar{P}_{m}^{t h}$ is the maximum missed detection probability at which the SUs interfere with PU. Then, the optimal frame duration is given as,

$$
\min \left(\bar{T}_{f}\right) \geq T_{f}^{t h} ; \text { such that: } \frac{\bar{C}}{C} \geq \beta, \frac{\bar{P}_{C}}{\bar{P}_{C}^{t h}} \geq \alpha, \beta, \alpha>1
$$

and:

$$
\begin{aligned}
& \bar{C}=\frac{1}{\bar{T}_{f}}\left(\bar{T}_{f}-\bar{T}_{s, i m a x}-T_{r}\right) \bar{P}_{C} P_{o} \\
& C=\frac{1}{T_{f}}\left(T_{f}-T_{s}-M T_{r}\right) \bar{P}_{C}^{t h} P_{o}
\end{aligned}
$$

Then, divide $\bar{C}$ by $C$, and after mathematical manipulations we obtain:

$$
T_{f}^{t h}=\frac{\alpha T_{f}\left(\bar{T}_{s, i m a x}+T_{r}\right)}{(\alpha-\beta) T_{f}+\beta\left(T_{s}-M T_{r}\right)}
$$

If $\alpha \approx \beta$, then

$$
T_{f}^{t h} \approx \frac{T_{f}\left(\bar{T}_{s, \text { imax }}+T_{r}\right)}{\left(T_{s}-M T_{r}\right)}
$$

Hence, 


$$
\min \left(\bar{T}_{f}\right) \geq \frac{T_{f}\left(\bar{T}_{s, i m a x}+T_{r}\right)}{\left(T_{s}-M T_{r}\right)}, M \ll \frac{T_{s}}{T_{r}}
$$

and the same for $m$ users:

$$
\min \left(\overline{\bar{T}}_{f}\right) \geq \frac{T_{f}\left(\overline{\bar{T}}_{s, i m a x}+T_{r}\right)}{\left(T_{s}-m T_{r}\right)}, m \ll \frac{T_{s}}{T_{r}}
$$

Then, $\bar{T}_{t}$ can be defined as: $\bar{T}_{t}=\bar{T}_{f}-\bar{T}_{s, i m a x}-T_{r}$, and $\bar{C}=\frac{\bar{T}_{t}}{\bar{T}_{f}} \bar{P}_{u}$, where $\bar{P}_{u}$ is the probability of having the final decision of unused under the proposed scheme denoted as $\bar{P}_{u}=P_{o} \bar{P}_{C}$.

The proposed frame structure for $M$ and $m$ users is depicted as in Figure 2(b), (c) it shows that: i) if $M$ users perform crossed sensing-reporting process (the worst case), then $\bar{T}_{s, i m a x}=T_{s}$, the maximum proposed sensing time equals to the sensing time in classical scheme, ii) both sensing and reporting time slots are crossed, which results in a longer transmission time $\left(\bar{T}_{t}\right)$ and in energy saving, and iii) the idle time slot is included in the crossed sensing-reporting time slot.

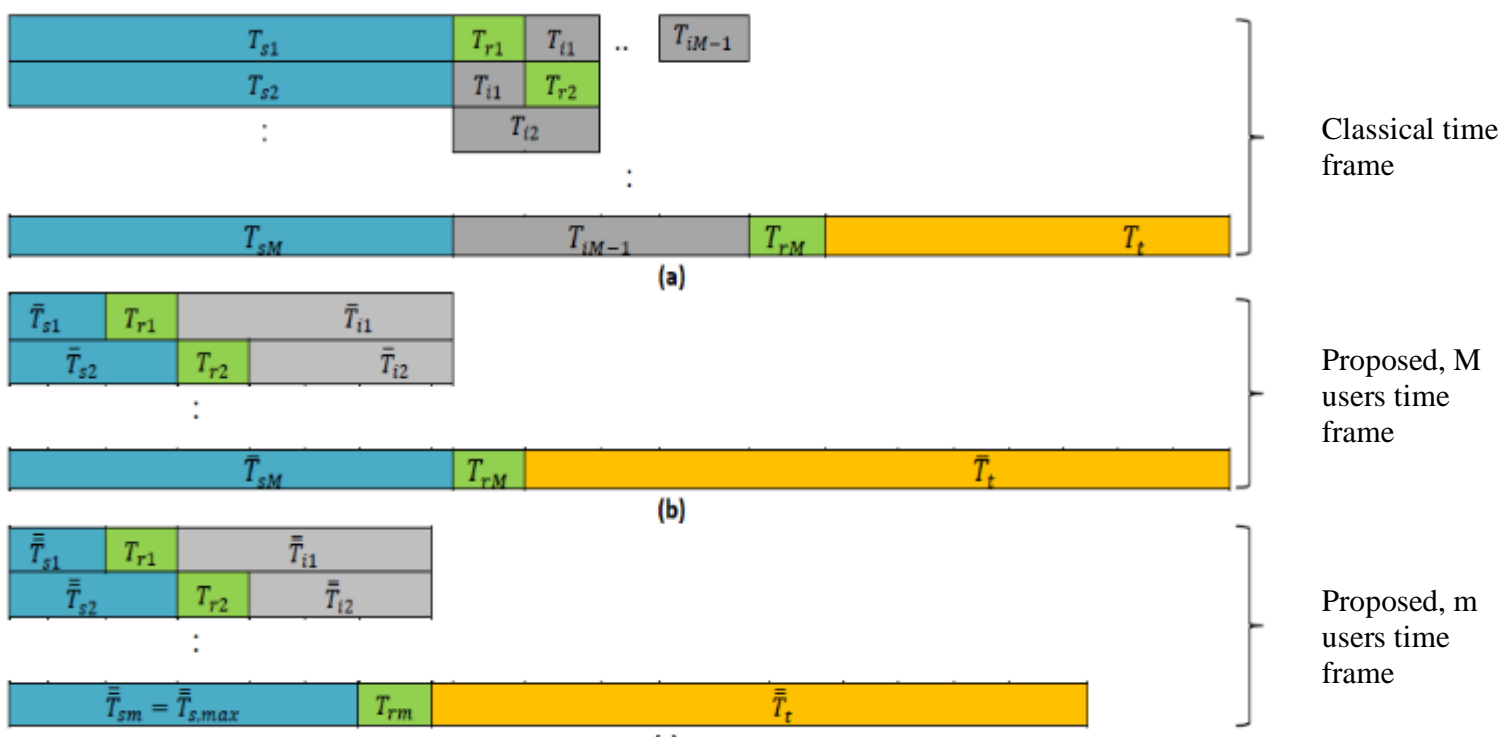

Figure 2. A comparison between classical and proposed frame structure; (a) classical time frame,

(b) proposed, $\mathrm{M}$ users time frame, (c) proposed, m users time frame

Hence, the saving in time consumption during the idle time, which eventually contributes to the overall energy saving, can be found from (13), (14) as a comparison between the proposed scheme with $M$ users versus the classical systems with $m$ users respectively as (19),

$$
\bar{\Delta} T_{\text {idle }}=\bar{T}_{i}-T_{i}=\left\{M \bar{T}_{s, i \max }-\sum_{i=1}^{M-1}\left(\bar{T}_{s, i}+T_{r}\right)\right\}-\left\{\left(M^{2}-M\right) T_{r}\right\}
$$

and

$$
\overline{\bar{\Delta}} T_{\text {idle }}=\overline{\bar{T}}_{i}-T_{i}=\left\{m \overline{\bar{T}}_{s, \text { max }}-\sum_{i=1}^{m-1}\left(\overline{\bar{T}}_{s, i}+T_{r}\right)\right\}-\left\{\left(M^{2}-M\right) T_{r}\right\}
$$

\subsection{Consumed energy}

Energy saving can be recognized by comparing the proposed and classical scheme. The total consumed energy during each frame in the proposed scheme with $M$ users is given by (21), 


$$
\bar{E}_{\text {tot }}=\sum_{i=1}^{M}\left(p_{s} \bar{T}_{s, i}+p_{r} T_{r}+p_{t} \bar{T}_{t} \bar{P}_{u}\right)
$$

and for $m$ users, it is given by (22),

$$
\overline{\bar{E}}_{t o t}=\sum_{i=1}^{m}\left(p_{s} \overline{\bar{T}}_{s, i}+p_{r} T_{r}\right)+\sum_{i=1}^{M} p_{t} \overline{\bar{T}}_{t} \bar{P}_{u}
$$

where $\overline{\bar{T}}_{t}=\overline{\bar{T}}_{f}-\overline{\bar{T}}_{s, i m a x}-T_{r}$. Hence, the overall energy saving in the proposed scheme, as compared with classical schemes, is achieved in two stages; first, a portion of energy is saved by performing sensing with unique time slots based on the SNR values at the ASUs' receivers. Second, a portion of energy is saved by preventing the SUs with SNR values below $S N R_{\text {wall }}$ from performing sensing and reporting processes. Then, the normalized energy saving in the first stage is given as (23),

$$
\bar{\Delta} \text { Energy }=\frac{E_{t o t}-\bar{E}_{t o t}}{E_{t o t}}
$$

and in the second stage it is given as (24)

$$
\overline{\bar{\Delta}} \text { Energy }=\frac{E_{t o t}-\overline{\bar{E}}_{t o t}}{E_{t o t}}
$$

The energy efficiency of both stages in the proposed scheme can be defined as the ratio between the consumed energy during data transmission and the overall consumed energy during each frame duration as in (25),

$$
\begin{aligned}
& \bar{\eta}_{E}=\frac{\sum_{i=1}^{M} \bar{P}_{u} p_{t} \bar{T}_{t}}{\sum_{i=1}^{M}\left(p_{s} \bar{T}_{s, i}+p_{r} T_{r}+p_{t} \bar{T}_{t} \bar{P}_{u}\right)} \\
& \overline{\bar{\eta}}_{E}=\frac{\sum_{i=1}^{m} \bar{P}_{u} p_{t} \overline{\bar{T}}_{t}}{\sum_{i=1}^{m}\left(p_{s} \overline{\bar{T}}_{s, i}+p_{r} T_{r}\right)+\sum_{i=1}^{M} p_{t} \overline{\bar{T}}_{t} \bar{P}_{u}}
\end{aligned}
$$

Finally, the proposed algorithm of cross sensing-reporting time can be summarized by using the pseudo code as shown below.

Proposed algorithm of cross sensing-reporting time BEGIN

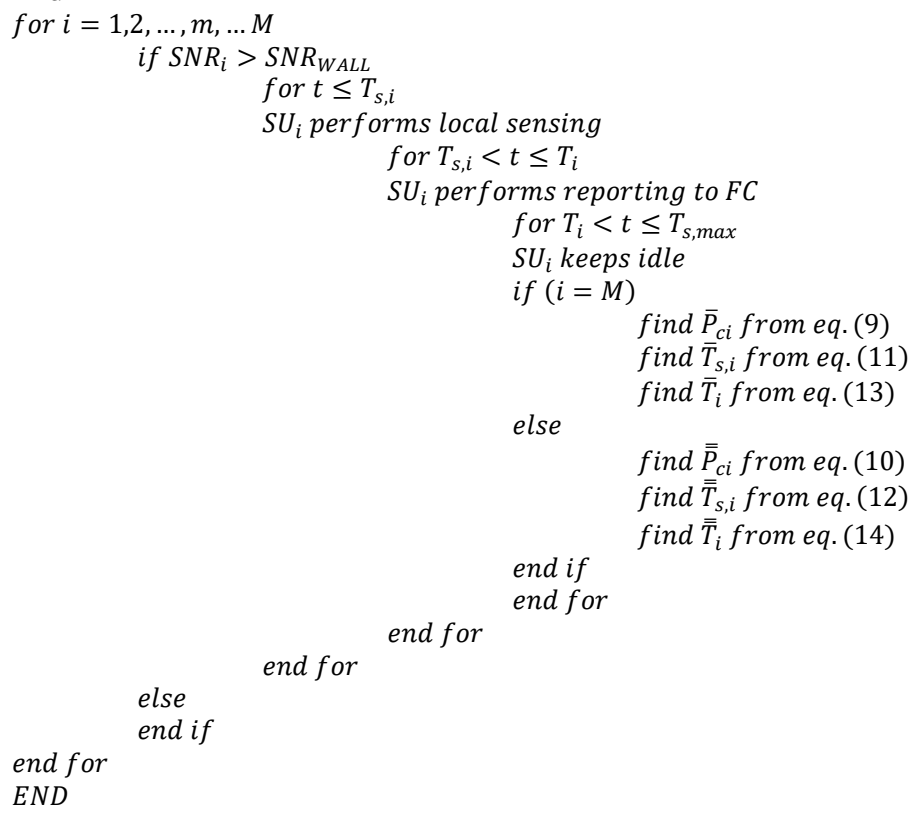




\section{NUMERICAL ANALYSIS AND PERFORMANCE EVALUATION}

Since the $S N R_{\text {wall }}$ does not have an exact value, and the frame time duration is limited, we considered that the $S N R_{\text {wall }}$ is the value of SNR that corresponds to a specific sensing time under the frame structure constraints. In this section, analytical results of the performance of the proposed scheme are presented and then compared with that of the classical scheme. Analytical parameters are shown in Table 1. (11), (12) show that the sensing time is exponentially decreasing with SNR.

Table 1. Analytical parameters

\begin{tabular}{cc}
\hline Parameter & Value \\
\hline $\bar{P}_{f}, \bar{P}_{d}$ & $0.05,0.8$ \\
$\Delta$ & 1.6 \\
$T_{r}$ & $0.5 \mathrm{~ms}$ \\
$p_{r}$ & $10 \mathrm{~mW}$ \\
$p_{t}$ & $10 \mathrm{~mW}$ \\
$p_{s}$ & $1 \mathrm{~mW}$ \\
$f_{s}$ & $0.5 \mathrm{MHz}$ \\
$P_{o}$ & 0.8 \\
\hline
\end{tabular}

Consider the $S N R_{\text {wall }}$ is $-22 \mathrm{~dB}$ [19], then SUs that receive signal less than $-22 \mathrm{~dB}$ are not allowed to perform sensing and reporting processes. Then, in both scenarios, 14 out of 20 SUs are performing sensing and reporting stages. Obviously, performing sensing of a received power with low SNR will not satisfy the targeted performance metrics. Also, it increases the sensing time slot. Figure 3 shows a comparison between the proposed and classical sensing time versus SNR. In the classical scheme, all SUs perform sensing processes synchronously during a specific time slot. In the first stage of the proposed scheme, each SU spends a sensing time according to the SNR level at each node, while in the second stage, the nodes with SNR lower than $S N R_{\text {wall }}$ are discarded from both sensing and reporting processes. This in turn increases the transmission time slot by decreasing the sensing time slot by about $62 \%$.

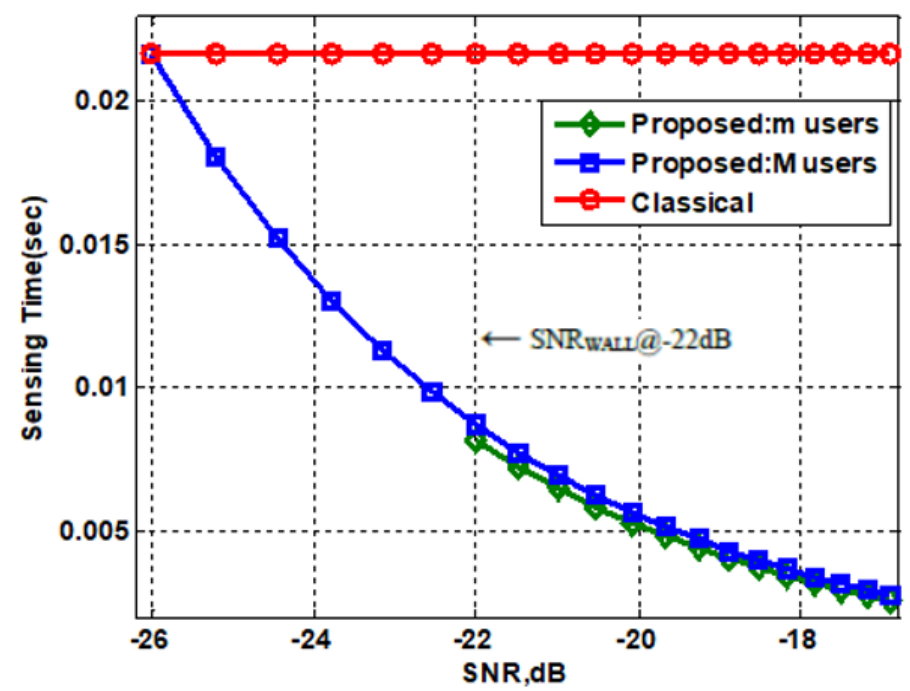

Figure 3. A comparison between proposed and classical sensing time versus $\mathrm{SNR}$ in $\mathrm{dB}$, when $\mathrm{m}$ out of $\mathrm{M}$ SUs perform sensing and reporting processes

A comparison between the achievable normalized throughput using different frame durations is depicted as shown in Figure 4. The enhanced achievable throughput is constrained by the frame duration. As $\bar{T}_{f}<T_{f}^{t h}$, the throughput of the proposed scheme is lower than that in the classical scheme for all SNR values less than $-19.5 \mathrm{~dB}$. When $\bar{T}_{f}=T_{f}^{t h}$, the achievable throughput matches the classical one at about $-22 \mathrm{~dB}$. Then, the throughput of the proposed scheme overcomes that of the classical one dramatically as $\bar{T}_{f}>T_{f}^{t h}$. 


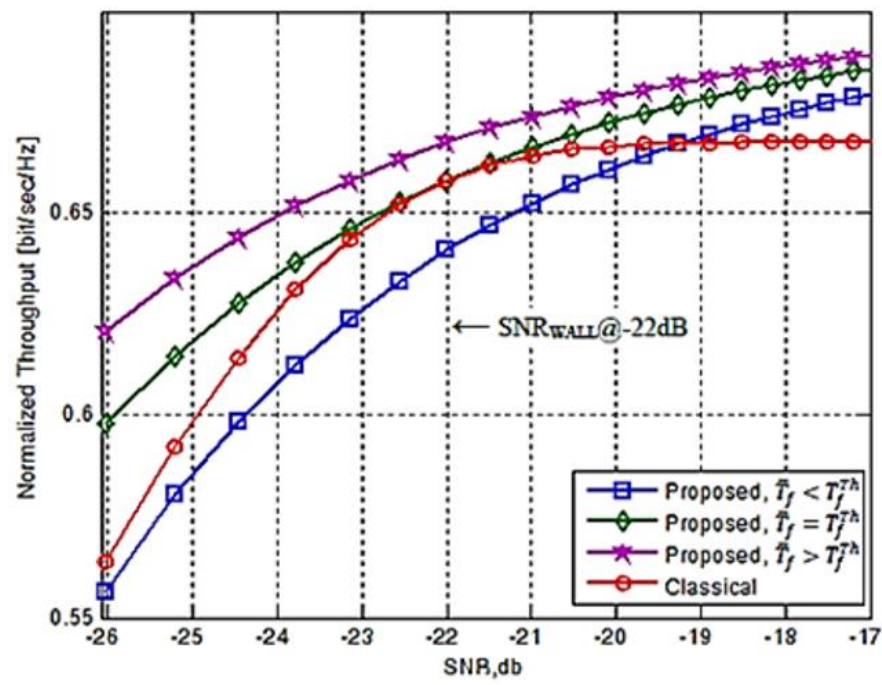

Figure 4. Normalized throughput versus SNR in dB using different frame durations

As mentioned earlier, saving the consumed energy during sensing and reporting is attained by two saving stages; by implementing asynchronous crossed sensing-reporting of local decisions, and by discarding some SUs from performing these duties. Figure 5 compare the proposed and classical schemes in terms of accumulated consumed energy during the sensing process. As shown, the accumulated consumed energy in the classical scheme is monotonically increasing. When the first stage of the asynchronous crossed sensingreporting is implemented for $20 \mathrm{SUs}$, the consumed energy drops from $0.15 \mathrm{~mJ}$ to $0.1 \mathrm{~mJ}$ at $-22 \mathrm{~dB}$ in the classical scheme, while it is drops from $0.44 \mathrm{~mJ}$ to $0.16 \mathrm{~mJ}$ at $-17 \mathrm{~dB}$. Nevertheless, discarding SUs with SNR less than the $S_{N R}$ wall from sensing and reporting results in lowering the number of SUs to 14 . This leads to decrease the consumed energy to about $0.018 \mathrm{~mJ}$ at $-22 \mathrm{~dB}$ and to $0.052 \mathrm{~mJ}$ at $-17 \mathrm{~dB}$.

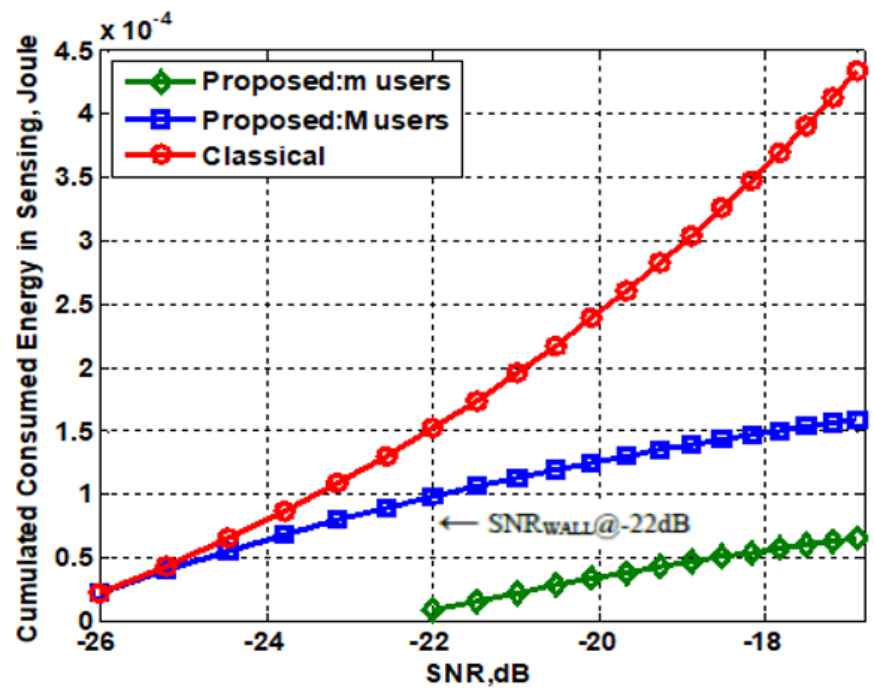

Figure 5. Cumulated sensing time during frame duration versus SNR in dB: A comparison between classical and the two-stages proposed scheme

The energy efficiency of this study is defined as the ratio between consumed energy during data transmission to the total consumed energy in the total frame duration. Figure 6 shows that the two-stages proposed scheme achieves high system efficiency at both low and high SNR. In the first stage of the proposed scheme, the system efficiency outperforms the classical one by $4.8 \%$, and by $5.2 \%$ in the second stage at $-17 \mathrm{~dB}$. It also achieves $100 \%$ energy efficiency for all SUs with SNR values less than $S N R_{\text {wall }}$. 


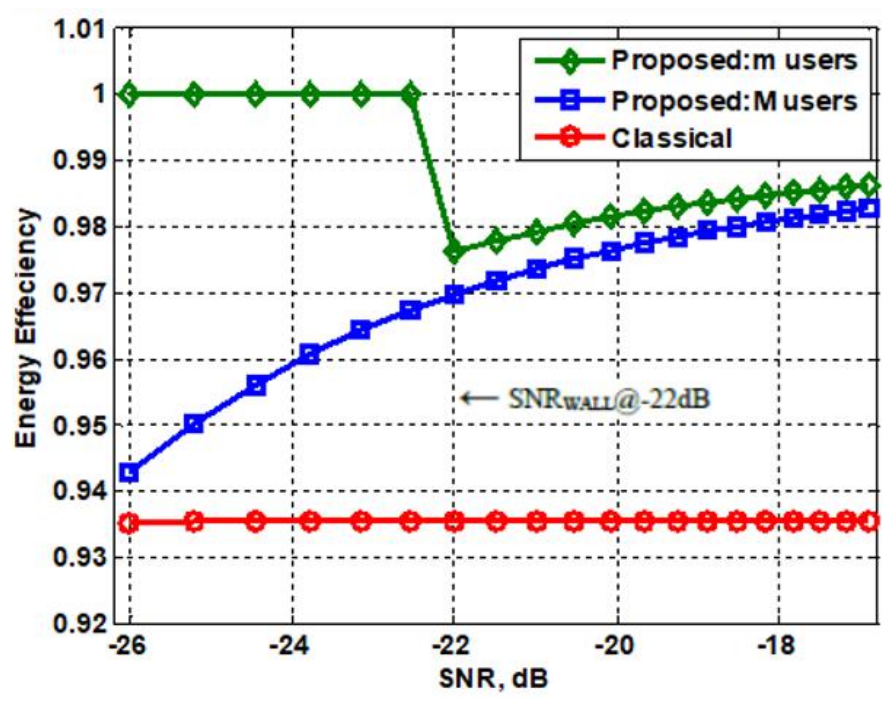

Figure 6. System energy efficiency: A comparison between classical and the two-stages proposed scheme versus $\mathrm{SNR}$ in $\mathrm{dB}$

The frame duration is divided into sensing, reporting, idle and transmission time slots. Energy is consumed in sensing, reporting and transmission time slots. Figures 7 and 8 provide a comparison between the proposed and classical schemes in terms of time slots and consumed energy divisions during one frame duration. The percentage of time slots division for the proposed and classical schemes is depicted in Figure 7. Under the same performance metrics in both schemes, the sensing time slot in the proposed scheme is dramatically reduced. This is achieved by exploiting the idle time during sensing process and the remaining time is exploited for data transmission. In the proposed scheme, excluding some of SUs from performing sensing and reporting will lead to reducing the sensing and idle time slots and gain a longer transmission time slot. As a numerical comparison with the classical scheme and during one frame duration, the sensing slot decreased from $27 \%$ of the frame duration to $10 \%$ and $6 \%$ for $\mathrm{M}$ and $\mathrm{m}$ users, respectively. The transmission slot increased from $60 \%$ of the total frame duration to $72 \%$ and $89 \%$ for $\mathrm{M}$ and $\mathrm{m}$ users, respectively.

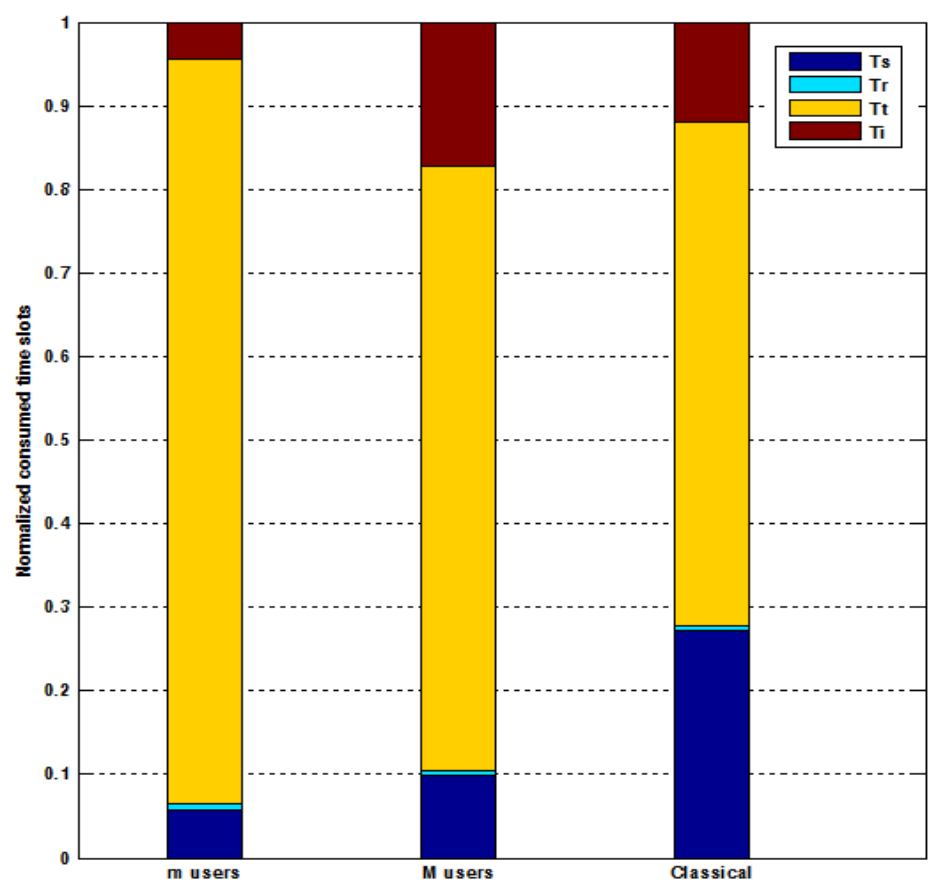

Figure 7. Normalized consumed time slot: A comparison between proposed and classical schemes 


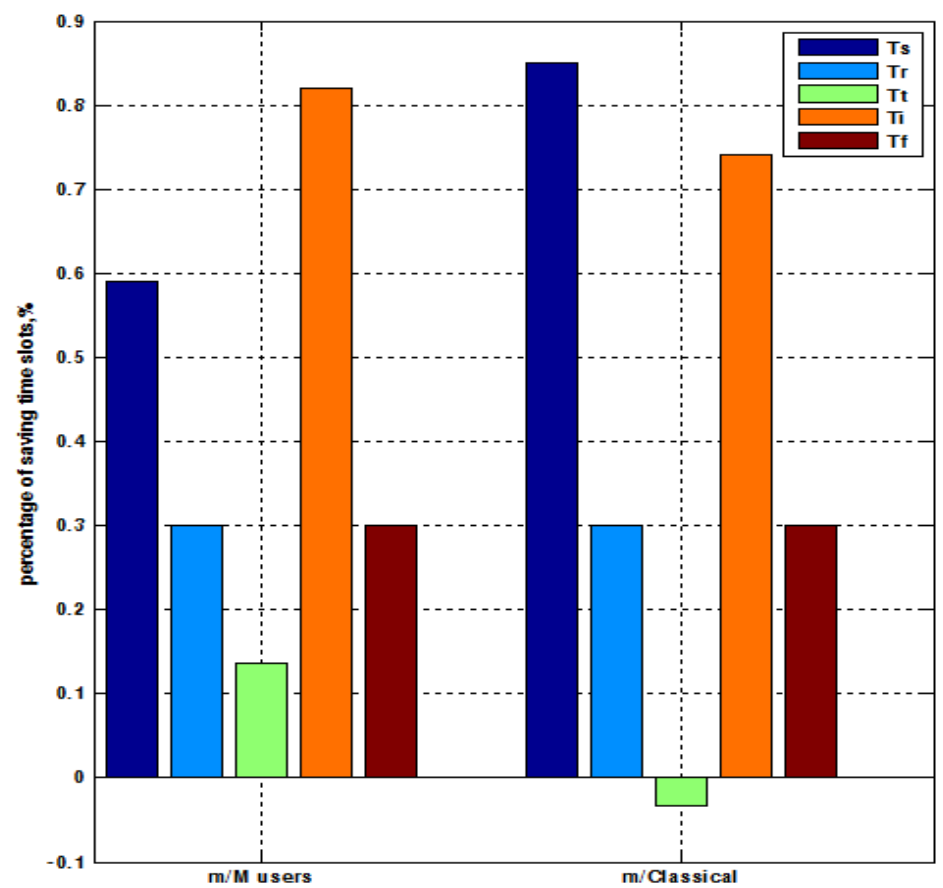

Figure 8. Time frame structure and time slot divisions and saving in the proposed scheme as a comparison with the classical scheme

Figure 8 depicts the saving in time slots in the proposed second stage as compared with the first proposed stage and the classical scheme. In this stage, the number of SUs performing sensing and reporting is reduced from 20 to 14 users. The rest discarded SUs are characterized with the lowest SNR, and those SUs are responsible for adopting a persistent sensing time slot by the FC in order to accommodate the predefined performance metrics. Also, this stage outperforms both of the first stage and the classical scheme by saving $30 \%$ of the reporting time slot, which reflected on reducing the total frame duration by $30 \%$. The decrement in total frame duration comes from saving in sensing time by $59 \%, 85 \%$ and $82 \%, 74 \%$ in the idle time as compared with first stage and classical scheme, respectively. As a result, $14 \%$ saving in transmission time is achieved as compared with the first stage, while the transmission time increased by $3 \%$ as compared with the classical scheme.

\section{CONCLUSION}

This paper proposed a scheme of an asynchronous and crossed sensing-reporting process in spectrum sensing. This scheme based on the following points: i) utilize the idle time slot in the reporting time slot, ii) allocate different sensing time for each SU based on the level of the SNR at each node, iii) discard some SUs from performing sensing and reporting processes based on the $S N R_{w a l l}$, and iv) optimize the total frame duration. The great benefits of this scheme can be summarized in three points: i) it's ability to attain saving in the consumed time and energy during sensing and reporting processes, ii) increase the efficiency of the CR system by increasing the transmission time slot within the frame duration, and iii) maintain the reliability of the uncertain communication environment within the acceptable performance metrics. A comparison between the proposed and classical schemes is numerically presented. The final results show that the proposed work is more efficient in terms of power consumption, throughput and reliability under noise uncertainty and low SNR environmental scenarios.

\section{REFERENCES}

[1] A. M. Arar, A. M. Masri, H. O. Ghannam, and I. K. Tumar, "A proposed scheme for dynamic threshold versus noise uncertainty in cognitive radio networks (DTNU)," Wireless Personal Communications, vol. 96, pp. 4543-4555, 2017, doi: 10.1007/s11277017-4402-1.

[2] K. Chabbra, G. Mahendru, and P. Banerjee, "Effect of dynamic threshold and noise uncertainty in energy detection spectrum sensing technique for cognitive radio systems," 2014 International Conference on Signal Processing and Integrated Networks (SPIN), 2014, pp. 377-361, doi: 10.1109/SPIN.2014.6776982. 
[3] S. Li, S. Xiao, M. Zhang, and X. Zhang, "Power saving and improving the throughput of spectrum sharing in wideband cognitive radio networks," in Journal of Communications and Networks, vol. 17, no. 4, pp. 394-405, Aug. 2015, doi: 10.1109/JCN.2015.000070.

[4] E. Lagunas and M. Nájar, "Spectral feature detection with sub-nyquist sampling for wideband spectrum sensing," in IEEE Transactions on Wireless Communications, vol. 14, no. 7, pp. 3978-3990, Jul. 2015, doi: 10.1109/TWC.2015.2415774

[5] F. Liu, J. Wang, and Y. Han, "An adaptive double thresholds scheme for spectrum sensing in cognitive radio networks," 2013 IEEE International Conference on Signal Processing, Communication and Computing (ICSPCC 2013), 2013, pp. 1-5, doi: 10.1109/ICSPCC.2013.6664104.

[6] P. Ren, J. Wang, and S. Li, "A dynamic spectrum access network based on cognitive radio," Journal of Electronics (China), vol. 27, pp. 577-610, 2010, doi: 10.1007/s11767-011-0622-9.

[7] A. Roozgard, Y. Tachwali, N. Barzigar, and S. Cheng, "Collaborative spectrum sensing for cognitive radio networks," IntechOpen, 2012, doi: 10.5772/32226.

[8] J. Ren, N. Zhang, and X. Shen, "Energy-efficient spectrum management for cognitive radio sensor networks," Springer Science and Business Media LLC, 2018, doi: 10.1007/978-3-319-60318-6.

[9] D. T. Dagne, K. A. Fante, and G. A. Desta, "Compressive sensing based maximum-minimum subband energy detection for cognitive radios," Heliyon, vol. 6, no. 9, 2020, doi: 10.1016/j.heliyon.2020.e04906.

[10] S. Althunibat, R. Palacios, and F. Granelli, "Energy-efficient spectrum sensing in cognitive radio networks by coordinated reduction of the sensing users," 2012 IEEE International Conference on Communications (ICC), 2012, pp. 1399-1404, doi: 10.1109/ICC.2012.6363804

[11] D. Lekomtcev and R. Marsalek, "Evaluation of Kolmogorov-Smirnov test and energy detector techniques for cooperative spectrum sensing in real channel conditions," Telfor Journal, vol. 7, no. 1, pp. 31-36, 2015, doi: 10.5937/telfor1501031L.

[12] A. El-Saleh, M. Ismail, M. Ali, M. Kamarudin, and T. Rahman, "analytical simulation and performance optimization for spectrum sensing in cognitive radio networks," International Journal on Computer Science and Engineering (IJCSE), vol. 3 no. 2, pp. 554-568, 2011

[13] Y. Liang, Y. Zeng, E. C. Y. Peh, and A. T. Hoang, "Sensing-throughput tradeoff for cognitive radio networks," in IEEE Transactions on Wireless Communications, vol. 7, no. 4, pp. 1326-1337, Apr. 2008, doi: 10.1109/TWC.2008.060869.

[14] Y. Liu, R. Yu, S. Xie, Y. Zhang, and V. C. M. Leung, "Efficient spectrum discovery with energy constraints in cognitive radio networks, “ 2011 18th International Conference on Telecommunications, 2011, pp. 38-43, doi: 10.1109/CTS.2011.5898956.

[15] K. Hamdi and K. Ben Letaief, "Power, sensing time, and throughput tradeoffs in cognitive radio systems: A cross-layer approach," 2009 IEEE Wireless Communications and Networking Conference, 2009, pp. 1-5, doi: 10.1109/WCNC.2009.4917914.

[16] G. Mahendru, A. Shukla, and P. Banerjee, "A novel mathematical model for energy detection based spectrum sensing in cognitive radio networks," Wireless Personal Communications, vol. 110, pp. 1237-1249, 2019, doi: 10.1007/s11277-019-06783-3.

[17] G. Mahendru, A. K. Shukla, and L. M. Patnaik, "A metaheuristic based approach for threshold optimization for spectrum sensing in cognitive radio networks," Recent Patents on Engineering, vol. 14, pp. 579-587, 2020, doi: 10.2174/1872212113666191025130543.

[18] K. M. Captain and M. V. Joshi, "SNR wall for cooperative spectrum sensing using generalized energy detector," 2018 10th International Conference on Communication Systems and Networks (COMSNETS), 2018, pp. 143-150, doi: 10.1109/COMSNETS.2018.8328191.

[19] R. Tandra and A. Sahai, "SNR walls for signal detection," in IEEE Journal of Selected Topics in Signal Processing, vol. 2, no. 1, pp. 4-17, Feb. 2008, doi: 10.1109/JSTSP.2007.914879.

[20] S. Maleki, S. P. Chepuri, and G. Leus, "Energy and throughput efficient strategies for cooperative spectrum sensing in cognitive radios," 2011 IEEE 12th International Workshop on Signal Processing Advances in Wireless Communications, 2011, pp. 71-75, doi: 10.1109/SPAWC.2011.5990482

[21] D. Chen, J. Li, and J. Ma, "Cooperative spectrum sensing under noise uncertainty in cognitive radio," 2008 4th International Conference on Wireless Communications, Networking and Mobile Computing, 2008, pp. 1-4, doi: 10.1109/WiCom.2008.295.

[22] S. Maleki, S. P. Chepuri, and G. Leus, "Optimization of hard fusion based spectrum sensing for energy-constrained cognitive radio networks," Physical Comm., vol. 9, pp. 193-198, 2013, doi: 10.1016/j.phycom.2012.07.003.

[23] J. Shen, S. Liu, R. Zhang, and Y. Liu, "Soft versus hard cooperative energy detection under low SNR," Third Int. Conf. on Comm. Net. in China, 2008, pp. 128-131, doi: 10.1109/CHINACOM.2008.4684985.

[24] T. Tian, H. Iwai, and H. Sasaoka, "Pseudo BER based SNR estimation for energy detection scheme in cognitive radio," IEEE 75th Vehicular Technology Conference, 2012, pp. 1-5, doi: 10.1109/VETECS.2012.6240226.

[25] M. Hamid and N. Björsell, "A novel approach for energy detector sensing time and periodic sensing interval optimization in cognitive radios," Proceedings of the 4th International Conference on Cognitive Radio and Advanced Spectrum Management, 2011, pp. 1-7, doi: 10.1145/2093256.2093314.

[26] C. Yao, Q. Wu, and L. Zhou, "Traffic based optimization of spectrum sensing in cognitive radio networks," Mathematical Problems in Engineering, vol. 2014, 2014, Art. no. 34935010, doi: 1155/2014/349350.

[27] Z. Ye, G. Memik, and J. Grosspietsch, "Energy detection using estimated noise variance for spectrum sensing in cognitive radio networks, “2 2008 IEEE Wireless Communications and Networking Conference, 2008, pp. 711-716, doi: 10.1109/WCNC.2008.131.

[28] S. Althunibat and F. Granelli, "Novel energy-efficient reporting scheme for spectrum sensing results in cognitive radio," IEEE Int. Conf. on Communications (ICC), 2013, pp. 2438-2442, doi: 10.1109/ICC.2013.6654897.

[29] S. Althunibat, S. Narayanan, M. Di Renzo, and F. Granelli, "Energy-efficient cooperative spectrum sensing for cognitive radio networks," IGI Global, 2015, doi: 10.4018/978-1-4666-6571-2.CH004

[30] G. P. Aquino, D. A. Guimarães, L. L. Mendes and T. C. Pimenta, "Combined pre-distortion and censoring for bandwidth-efficient and energy-efficient fusion of spectrum sensing information," Sensors, vol. 17, 2017, Art. no. 654, doi: 10.3390/s17030654.

[31] S. Althunibat and F. Granelli, "Energy efficiency analysis of soft and hard cooperative spectrum sensing schemes in cognitive radio networks," 2014 IEEE 79th Vehicular Technology Conference (VTC Spring), 2014, pp. 1-5, doi: 10.1109/VTCSpring.2014.7022904.

[32] X. Cai, X. Chen, H. Chen, and J. Liu, "Step combination cooperative spectrum sensing algorithm based on autocorrelation matrix," 2011 Global Mobile Congress, 2011, pp. 1-5, doi: 10.1109/GMC.2011.6103924.

[33] H. Lee, Y. Ko, S. Vahid, and K. Moessner, "Practical spectrum aggregation for secondary networks with imperfect sensing," in IEEE Transactions on Vehicular Technology, vol. 65, no. 7, pp. 5474-5484, July 2016, doi: 10.1109/TVT.2015.2465388.

[34] S. Bhattacharjee, R. Rajkumari, and N. Marchang, "Effect of colluding attack in collaborative spectrum sensing," 2nd Int. Conf. on Signal Processing and Integrated Net., 2015, pp. 223-227, doi: 10.1109/SPIN.2015.7095266.

[35] A. Taherpour, H. Mokhtarzadeh, and T. Khattab, "Optimized error probability for weighted collaborative spectrum sensing in time-and energy-limited cognitive radio networks," in IEEE Transactions on Vehicular Technology, vol. 66, no. 10, pp. 9035-9049, Oct. 2017, doi: 10.1109/TVT.2017.2706142. 
[36] G. Mahendru, A. K. Shukla, P. Banerjee, and L. M. Patnaik, "Adaptive double threshold-based spectrum sensing to overcome sensing failure in presence of noise uncertainty," 2019 6th International Conference on Signal Processing and Integrated Networks (SPIN), 2019, pp. 466-471, doi: 10.1109/SPIN.2019.8711570.

\section{BIOGRAPHIES OF AUTHORS}

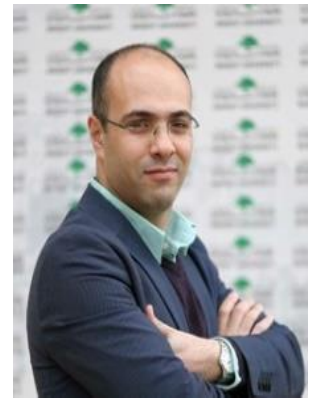

Iyad Khalil Tumar (D) SC SC $\mathrm{P}$ is an Assistant Professor at Electrical and Computer Engineering Department at Birzeit University (BZU). He is currently a project manager for two research projects funded by Qatar Foundation and BMBF in Germany. He received his bachelor degree in electrical engineer (communication systems) in 2002, master degree in computational science in 2006 from BZU in Palestine, and he received his doctoral (Ph.D.) degree in smart systems in 2010 from Jacobs University Bremen at Germany. He was a research associate and a member of computer networks and distributed systems group at Jacobs University Bremen. His research interests are resource management of disruption tolerant networks, wireless communication, wireless sensor networks, underwater networks, and network management. He was a research associate and a member of EMANICS from 2007-2010. Iyad Tumar is a reviewer for IEEE/ACM journals and conferences. He can be contacted at email: itumar@birzeit.edu.

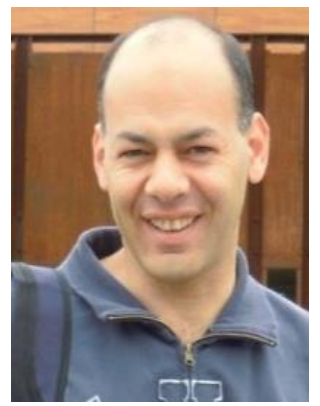

Adnan Mohammad Arar (iD SI Sc P received his B.Sc. degree in Electronics Engineering from Al-Quds University, Palestine, in 2001. His Specialized Diploma in Radiation Protection from HIAST Institute, Syria, in 2003. And his Master degree in Telecommunications Engineering from Universita Degli Studi Di Trento, Italy, in 2012. Currently, he works a lecturer in the College of Science and Technology at Hebron University. His research interests include cognitive radio: spectrum sensing, energy efficiency and throughput optimization. He can be contacted at email: adnana@hebron.edu.

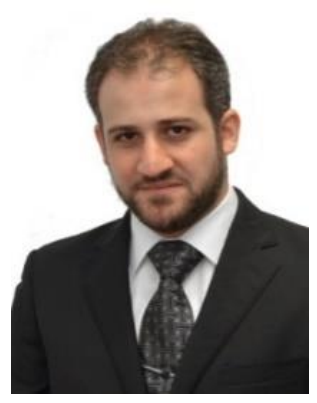

Ayman Abd El-Saleh (iD 8 SC $\mathrm{P}$ received his B.Sc. degree in Communications Engineering from Omar El-Mukhtar University (OMU), Libya, in 1999, his M.Sc. in Microelectronics Engineering and his Ph.D. in Wireless Communications both from Universiti Kebangsaan Malaysia (UKM), in 2006 and 2012, respectively. He joined the Faculty of Engineering at Multimedia University (MMU) in October 2006 at which he was a Senior Lecturer and the Chairperson of Centre for Wireless Technology (CWT). In April 2017, he was appointed as the Lead of Connectivity Research Theme at MMU. He joined A'Sharqiyah University in Oman in September 2017 at which he is currently an Assistant Professor. He is also a Senior Member of IEEE, and was an Executive Committee Member of IEEE Malaysia ComSoc/VTS Joint Chapter in 2015 and 2016. He is a reviewer of several ISI-indexed journals and published about 60 journal and conference articles. His research interests include cognitive radio networks, heterogeneous LTE/LTE-advanced cellular networks, and applications of artificial intelligence and evolutionary algorithms in wireless communications. He can be contacted at email: ayman.elsaleh@asu.edu.om. 